\title{
L'irrigation en France depuis 1988
}

\author{
par Jean-Louis Janin
}

ICGREF,

Chargé du pôle de compétence statistique

du réseau IFEN-DIREN pour le Sud-Ouest

\section{I $41 \%$ D'AUGMENTATION EN SEPT ANS}

La superficie totale irriguée en France métropolitaine a augmenté de 470000 ha entre 1988 et 1995 . En passant de 1,15 à 1,62 million d'hectares elle augmente de $41 \%$ en sept ans, soit une augmentation moyenne de $5 \%$ par an.

La connaissance statistique de l'irrigation en France métropolitaine remonte à plus d'un siècle [1]. La superficie irriguée a été multipliée par quatre en 40 ans, de 1955 à 1995. La quasi stagnation que semblent faire apparaître les estimations de 1990 et 1993 est démentie par l'augmentation d'environ 150000 ha entre 1993 et 1995 .

Tableau 1. - Superficies irriguées de 1955 à 1995.

\begin{tabular}{|c|c|c|}
\hline Année & 1000 ha & Source \\
\hline 1955 & 402 & INSEE (RGA 55) \\
\hline 1970 & 539 & SCEES (RGA 70) \\
\hline 1979 & 801 & SCEES (RGA 79) \\
\hline 1988 & 1147 & SCEES (RGA 88) \\
\hline 1990 & 1476 & SCEES (STRU 90) \\
\hline 1993 & 1468 & SCEES (STRU 93) \\
\hline 1995 & 1620 & SCEES (STRU 95) \\
\hline
\end{tabular}

Il s'agit bien d'une tendance de fond de l'agriculture française, analysée ici sur la période récente, durant laquelle la nouvelle politique agricole commune a été définie et mise en œuvre.

L'étude statistique porte sur l'ensemble du territoire métropolitain et sur les découpages géographiques des 6 bassins des Agences de l'Eau, des 22 régions et des 96 départements.

Pour assurer une qualité statistique homogène aux données présentées, le traitement des résultats bruts d'enquêtes effectuées par le SCEES (Service central des enquêtes et études statistiques) a été complété par un réquilibrage des valeurs extrapolées et par une sélection des entités géographiques renseignées (encadré).
Cette étude a été rendue possible par la mise à disposition des fichiers source et grâce à une collaboration très fructueuse avec les responsables et les spécialistes du SCEES tout au long du processus d'élaboration de la méthode d'analyse et de restitution des données, engagé depuis octobre 1994 [2].

\section{Une information à géographie variable}

L'information présentée ici provient du recensement agricole de 1988 et des enquêtes sur la structure des exploitations agricoles de 1990, 1993 et 1995, conduits

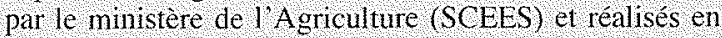
département, avec une coordination régionale, par les services statistiques des DDAF (Directions départementales de l'agriculture) et des DRAF (Directions régionales de l agniculture et de la forêt). Le seuil d'échan tillomage des données d'enquête restituees ici a été fixé a 30 exploitations, avec un plafond de $35 \%$ pour le réquilibrage des données également recuelllies en 1988.

Ces contraintes limitent le nombre de départements, de régions ou de bassins renseignés pour chaque donnée traitée Le rapport des valeurs obtenues sur le territoire renseigne et sur 1 ensemble du territoire foumit un taux de localisation de la donnée dans le découpage de la carte.

L'instruction administrative des aides de la politique agricole commune aux cultures de céréales, oléagineux et protéagineux apporte une information complémentaire pour 1995. Les domnées présentees ici sont issues d un traitement statistique provisoire de données individuelles issues des fichiers constitués par 1 ONIC (Office national interprofessionnel des céreales) pour liquider les demandes $\mathrm{d}$ aides recueillies en département (superficies déclarées par commune de localisation des parcelles).

\section{II $90 \%$ DES IRRIGANTS REGROUPÉS} DANS 3 BASSINS

Les bassins Adour-Garonne, Loire-Bretagne et Rhône-Méditerrannée-Corse totalisent par alleurs $88 \%$ des superficies irriguées en 1995. 
Une analyse plus détaillée montre que le nombre d'exploitations qui irriguent a diminué de $8.4 \%$ entre 1988 et 1995, ce qui est nettement plus faible que la diminution globale du nombre d'exploitations agricoles, évalué sur les mêmes bases à $27 \%$. Les deux phénomènes de l'introduction de l'irrigation dans de nouvelles exploitations et d'une meilleure résistance de celles qui irriguent à la disparition ou à l'absorption se conjuguent ici.

Tableau 2. - Irrigation par bassin en 1988 et 1995.

\begin{tabular}{|l|r|r|r|r|}
\hline \multirow{2}{*}{ Bassin } & \multicolumn{2}{|c|}{1000 expl. } & \multicolumn{2}{c|}{1000 ha } \\
\cline { 2 - 5 } & 1988 & 1995 & 1988 & 1995 \\
\hline France & 121 & 110 & 1147 & 1620 \\
\hline Adour-Garonne & 40 & 38 & 505 & 661 \\
Loire-Bretagne & 21 & 21 & 263 & 414 \\
Rhône-Méd-Corse & 50 & 41 & 268 & 355 \\
Seine-Normandie & 5,3 & 5,9 & 69 & 110 \\
Rhin-Meuse & 2,6 & 2,7 & 34 & 50 \\
Artois-Picardie & 1,4 & 1,9 & 7,8 & 26 \\
\hline
\end{tabular}

Source : SCEES (RGA 88 et STRU 95).

La carte 1 présente la chronique des superficies irriguées par région pour les 4 années de données. La superficie irriguée en 1995 et la série des indices d'évolution sont fournies dans le tableau 4 (base 100 en 1988).

L'augmentation générale sur la période reste globalement marquée entre 1993 et 1995 (13 points d'augmentation de l'indice). Parmi les 7 régions où la superficie irriguée dépasse 100000 ha, l'indice augmente de 18 points en MidiPyrénées (qui frôle les 300000 ha irrigués), de 15 points en Aquitaine, et respectivement de 9,12 et 13 points en PoitouCharentes, Pays de la Loire et Rhône-Alpes.

Carte 1. - Irrigation en région de 1988 à 1995.

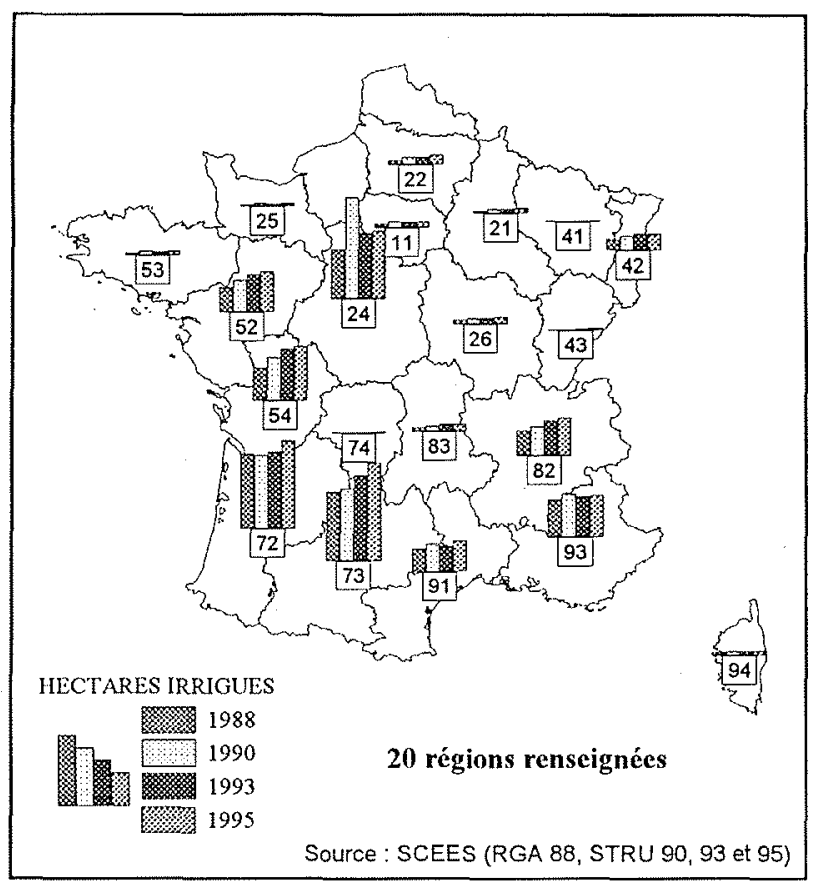

La région Centre a comnu un pic très marqué en 1990 (plus du double de la superficie irriguée en 1988), avec une forte diminution en 1993 et une augmentation de 5 points en fin de période. La région PACA connait une augmentation de 3 points, avec l'indice le moins élevé sur la période (111), qu'elle partage avec la Corse, dont l'indice perd 14 points entre 93 et 95 .

Dans les autres régions, l'augmentation reste très marquée, notamment en Languedoc-Roussillon $(+20)$, en Picardie $(+49)$ et en Bourgogne $(+33)$, sauf en Alsace, où l'indice perd 3 points.

L'analyse par bassin montre la persistance de l'augmentation en fin de période dans les bassins Adour-Garomne, Rhône-Méditerrannée-Corse et Seine-Normandie $(+15,+11$ et +15 ), le tassement relatif en Loire-Bretagne $(+8)$, la diminution dans le bassin Rhin-Meuse $(-3)$ et la poursuite du décollage spectaculaire dans le bassin Artois-Picardie $(+100)$

Tableau 3. - Superficies irriguées en 1995 et indices d'évolution depuis 1988 par bassin.

\begin{tabular}{|l|r|r|r|r|}
\hline \multirow{2}{*}{ Bassin } & 1 000 ha & \multicolumn{3}{|c|}{ Indice 100 en 1988} \\
\cline { 2 - 5 } & 1995 & 1990 & 1993 & 1995 \\
\hline France & 1620 & 129 & 128 & 141 \\
\hline Adour-Garonne & 661 & 104 & 116 & 131 \\
Loire-Bretagne & 414 & 158 & 149 & 157 \\
Rhône-Méd.-C. & 355 & 118 & 121 & 132 \\
Seine-Normandie & 110 & 213 & 143 & 158 \\
Rhin-Meuse & 50 & 130 & 151 & 146 \\
Artois-Picardie & 26 & 252 & 224 & 334 \\
\hline
\end{tabular}

Source : SCEES (RGA 88 et STRU 95).

Tableau 4. - Superficies irriguées en 1995 et indices d'évolution depuis 1988 par région.

\begin{tabular}{|c|c|c|c|c|}
\hline \multirow{2}{*}{ Région } & \multirow{2}{*}{$\begin{array}{c}\text { 1000 ha } \\
\end{array}$} & \multicolumn{3}{|c|}{ Indice 100 en 1988} \\
\cline { 3 - 5 } & 1995 & 1990 & 1993 & 1995 \\
\hline 73 & 299 & 105 & 124 & 142 \\
\hline 72 & 269 & 98 & 102 & 117 \\
\hline 24 & 206 & 205 & 133 & 138 \\
\hline 54 & 166 & 132 & 159 & 168 \\
\hline 93 & 129 & 115 & 108 & 111 \\
\hline 52 & 123 & 127 & 149 & 161 \\
\hline 82 & 116 & 119 & 139 & 152 \\
\hline 91 & 92 & 119 & 110 & 130 \\
\hline 42 & 50 & 131 & 152 & 149 \\
\hline 22 & 29 & 183 & 182 & 231 \\
\hline 83 & 23 & 135 & 173 & 184 \\
\hline 26 & 22 & 127 & 128 & 161 \\
\hline 11 & 19 & 164 & 134 & 152 \\
\hline 53 & 14 & 189 & 168 & 206 \\
\hline 21 & 13 & 242 & 256 & 280 \\
\hline 94 & 12 & 118 & 125 & 111 \\
\hline 25 & 6,9 & 184 & 150 & 169 \\
\hline 43 & 4,9 & 359 & 534 & 673 \\
\hline 74 & 3,2 & 103 & 116 & 154 \\
\hline 41 & 0,7 & 126 & 115 & 117 \\
\hline Ens. & 1598 & 128 & 127 & 140 \\
\hline
\end{tabular}

Source : SCEES (RGA 88, STRU 90, 93 et 95). 


\section{UN TAUX D'IRRIGATION MOYEN DE $5,7 \%$}

Il s'agit ici du taux d'irrigation global de la superficie agricole utilisée du territoire métropolitain. Un tel taux est lié au territoire considéré (7,9\% pour les 67 départements de la carte, $12 \%$ pour le bassin Adour-Garonne, etc...).

Tableau 5. - Taux d'irrigants et d'irrigation en 1988 et 1995.

\begin{tabular}{|l|r|r|r|r|}
\hline \multirow{2}{*}{ Bassin } & \multicolumn{2}{|c|}{ en nb d'expl. } & \multicolumn{2}{c|}{ en superficie } \\
\cline { 2 - 5 } & 1988 & 1995 & 1988 & 1995 \\
\hline France & 12,0 & 15,1 & 4,0 & 5,7 \\
\hline Rhône-Médit.-C. & 22,4 & 25,7 & 6,5 & 8,5 \\
Adour-Garonne & 18,7 & 23,2 & 9,1 & 12 \\
Loire-Bretagne & 6,3 & 8,9 & 2,6 & 4,2 \\
Rhin-Meuse & 5,3 & 8,1 & 2,4 & 3,5 \\
Artois-Picardie & 3,3 & 6,8 & 0,5 & 1,8 \\
Seine-Normandie & 3,7 & 5,2 & 1,1 & 1,8 \\
\hline
\end{tabular}

Source : SCEES (RGA 88 et STRU 95) h

Carte 2. - Taux d'irrigation global en département (1995).

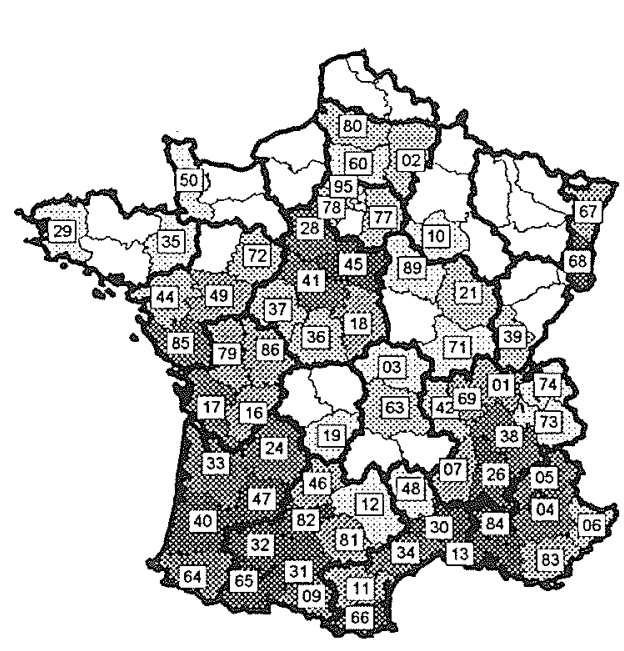

TAUX DIRRIGATION

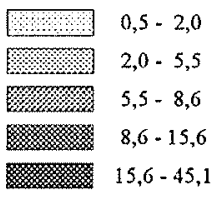

67 départements renseignés

Les limites de classes du taux d'irrigation visent à une répartition égale des départements (resp. 14

$14,13,13$ et 13 dans les classes numérotées de 1 à 5 vers le bas)

Source : SCEES (RGA 88 et STRU95)
Le taux d'irrigants (défini ici comme la proportion d'exploitations équipées pour l'irrigation sur l'ensemble des exploitations), est passé de $12 \%$ à $15,1 \%$ entre 1988 et 1995, soit une augmentation moyenne de 3,1\%.

Les bassins où l'augmentation est supérieure à la moyenne sont Adour-Garonne $(+4,5 \%)$, Rhône-Méditerrannée-Corse $(+3,3 \%)$ et Artois-Picardie $(+3,5 \%)$. Viennent ensuite les bassins Rhin-Meuse $(+2,8 \%)$, Loire-Bretagne $(+2,6 \%)$ et Seine-Normandie $(+1,6 \%)$

La carte 2 et le tableau 6 permettent de repérer les 13 départements dont le taux d'irrigation dépasse $15 \%$. Ils sont situés au cœur des quatre aires géographiques de développement de l'irrigation du Sud-Ouest (avec des prolongements en façade atlantique), du pourtour méditerrannnéen (avec des prolongements alpins), du bassin parisien (notamment le plateau de la Beauce) et de la plaine d'Alsace.

Tableau 6. - Taux d'irrigation et superficies irriguées par département (1995).

\begin{tabular}{|c|c|c|c|c|c|}
\hline Dép. & Taux & 1000 ha & Dép. & Taux & 1000 ha \\
\hline 13 & 45 & 69 & 18 & 6,4 & 29 \\
\hline 40 & 41 & 91 & 67 & 6,4 & 13 \\
\hline 68 & 26 & 37 & 46 & 5,7 & 13 \\
\hline 82 & 26 & 61 & 01 & 5,6 & 16 \\
\hline 47 & 25 & 82 & 07 & 5,5 & 8,8 \\
\hline 65 & 21 & 27 & 72 & 5,3 & 21 \\
\hline 26 & 20 & 44 & 37 & 5,0 & 17 \\
\hline 84 & 20 & 24 & 44 & 4,6 & 19 \\
\hline 32 & 19 & 91 & $2 A$ & 4,2 & 1,6 \\
\hline 30 & 17 & 32 & 42 & 3,5 & 8,6 \\
\hline 45 & 17 & 66 & 80 & 3,4 & 17 \\
\hline 31 & 16 & 60 & 78 & 3,3 & 3,2 \\
\hline 66 & 16 & 16 & 77 & 2,9 & 10 \\
\hline $2 B$ & 15 & 10 & 63 & 2,6 & 11 \\
\hline 33 & 15 & 40 & 36 & 2,5 & 11 \\
\hline 17 & 14 & 63 & 06 & 2,2 & 1,4 \\
\hline 05 & 13 & 12 & 02 & 2,0 & 10 \\
\hline 09 & 12 & 16 & 27 & 2,0 & 9,4 \\
\hline 41 & 12 & 38 & 39 & 2,0 & 3,8 \\
\hline 34 & 11 & 25 & 03 & 1,9 & 10 \\
\hline 04 & 10 & 16 & 10 & 1,9 & 7,6 \\
\hline 28 & 10 & 48 & 73 & 1,6 & 1,8 \\
\hline 38 & 9,5 & 25 & 29 & 1,5 & 6,2 \\
\hline 24 & 9,1 & 31 & 12 & 1,3 & 6,8 \\
\hline 64 & 8,9 & 32 & 60 & 1,3 & 4,9 \\
\hline 16 & 8,6 & 33 & 89 & 1,1 & 4,5 \\
\hline 85 & 8,6 & 41 & 35 & 0,9 & 4,3 \\
\hline 83 & 8,3 & 5,5 & 74 & 0,9 & 1,3 \\
\hline 81 & 8,1 & 26 & 95 & 0,8 & 0,5 \\
\hline 86 & 8,1 & 40 & 19 & 0,7 & 1,7 \\
\hline 49 & 8,0 & 39 & 48 & 0,5 & 1,4 \\
\hline 11 & 7,7 & 20 & 50 & 0,5 & 2,4 \\
\hline 69 & 7,2 & 11 & 71 & 0,5 & 2,7 \\
\hline 79 & 7,0 & 32 & Ens. & 7,9 & 1578 \\
\hline
\end{tabular}

Source: SCEES (STRU 95). 


\section{LES IRRIGANTS CULTIVENT $20 \%$ DU TERRITOIRE AGRICOLE}

La superficie totale cultivée par les exploitations équipées pour l'irrigation définit une SAU (Surface agricole utilisée) concernée par l'irrigation, c'est-à-dire dont l'assolement comporte des cultures permanentes ou annuelles irriguées (SAU \& I). Le taux correspondant est évalué à $20 \%$ de la SAU du territoire métropolitain.

L'obligation de disposer chaque année d'une autorisation administrative pour prélever de l'eau à titre individuel, au delà d'un certain seuil, et l'abaissement récent de ce seuil à $8 \mathrm{~m}^{3} / \mathrm{ha}$ dans les zones de pénurie de la ressource naturelle, soulignent la précarité de ce type de prélèvement.

Lorsqu'il y a réalimentation de la ressource sollicitée, il est fait obligation au demandeur de passer, avec un maître d'ouvrage public, une convention de restitution du débit prélevé. Cette restitution est opérée par le gestionnaire des ouvrages avec facturation du service rendu.

Il y aurait donc lieu de distinguer dans l'accès individuel à l'eau celui qui est garanti par une réalimentation à caractère collectif. Dans les mêmes conditions de réalimentation, la distribution de l'eau en réseau collectif prend alors un caractère doublement collectif.

Avec l'information disponible actuellement, la répartition de la SAU concernée est respectivement de $40 \%$ et $60 \%$, entre la SAU des exploitations alimentées en réseau collectif

Carte 3. - Superficie régionale cultivée par les irrigants et accès à l'eau (1995).

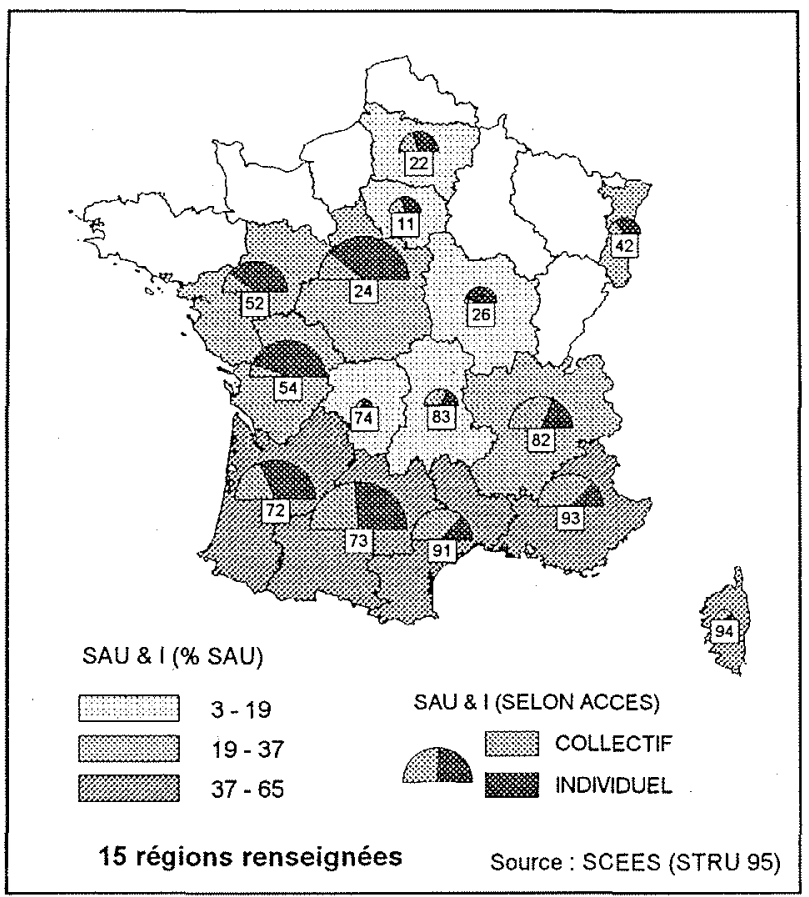

et celle des exploitations qui opèrent par prélèvement individuel direct.

Cette répartition est toutefois déséquilibrée au profit des réseaux collectifs, car la SAU des irrigants qui disposent des deux modes d'accès a été comptée en mode réseau collectif.

Tableau 7. - SAU des irrigants et accès à l'eau par bassin (1995).

\begin{tabular}{|l|c|c|c|c|}
\hline \multirow{2}{*}{\multicolumn{1}{c|}{ Bassin }} & \multicolumn{2}{c|}{ SAU \& 1} & \multicolumn{2}{c|}{$\%$ mode d'accès } \\
\cline { 2 - 5 } & $\%$ SAU & 1000 ha & réseau & indiv. \\
\hline France & 20 & 5600 & 40 & 60 \\
\hline Adour-Garonne & 35 & 1924 & 40 & 60 \\
Rhône-Méd.-C. & 31 & 1287 & 66 & 34 \\
Loire-Bretagne & 16 & 1548 & 21 & 79 \\
Seine-Normandie & 9,6 & 570 & 34 & 66 \\
Rhin-Meuse & 8,4 & 121 & 31 & 69 \\
\hline
\end{tabular}

Source : SCEES (STRU 95).

La SAU des irrigants en réseau collectif domine nettement au Sud-Est. La part des réseaux diminue en allant vers l'ouest. L'accès individuel prend le dessus en façade atlantique et en remontant vers le nord.

Tableau 8. - SAU des irrigants et accès à I'eau par départements (1995).

\begin{tabular}{|c|c|c|c|c|}
\hline \multirow{2}{*}{ Rég. } & \multicolumn{2}{|c|}{ SAU \& } & \multicolumn{2}{c|}{ Accès à I'eau (\%) } \\
\cline { 2 - 5 } & $\%$ SAU & 1000 ha & réseau & individuel \\
\hline 93 & 65 & 434 & 74 & 26 \\
\hline 72 & 42 & 637 & 38 & 62 \\
\hline 94 & 40 & 43 & 77 & 23 \\
\hline 73 & 39 & 919 & 48 & 52 \\
\hline 91 & 37 & 377 & 72 & 28 \\
\hline 42 & 33 & 114 & 29 & 71 \\
\hline 24 & 33 & 790 & 22 & 78 \\
\hline 54 & 32 & 560 & 12 & 88 \\
\hline 82 & 27 & 425 & 62 & 38 \\
\hline 52 & 19 & 416 & 20 & 80 \\
\hline 17 & 18 & 106 & 39 & 61 \\
\hline 22 & 12 & 164 & 41 & 59 \\
\hline 83 & 7,5 & 116 & 61 & 39 \\
\hline 26 & 6,3 & 113 & 9 & 91 \\
\hline 74 & 3,1 & 28 & 33 & 64 \\
\hline Ens. & 26 & 5241 & 41 & 59 \\
\hline
\end{tabular}

Source : SCEES (STRU 95). 


\section{UN TAUX D'IRRIGATION INTERNE DE $29 \%$}

Le taux moyen d'irrigation interne est évalué pour 1995 en France métropolitaine à $29 \%$ de la superficie agricole utilisée par les exploitations équipées pour l'irrigation.

Tableau 9. - Hectares irrigués et taux d'irrigation interne par bassin (1988-1995).

\begin{tabular}{|l|r|c|c|r|}
\hline \multirow{2}{*}{ Bassin } & \multicolumn{2}{|c|}{1 000 ha irrigués } & \multicolumn{2}{c|}{$\%$ (SAU \& I) } \\
\cline { 2 - 5 } & 1988 & 1995 & 1988 & 1995 \\
\hline France & 1147 & 1620 & 28 & 29 \\
\hline Adour-Garonne & 505 & 661 & 33 & 34 \\
Loire-Bretagne & 263 & 414 & 26 & 27 \\
Rhône-Méd.-C. & 268 & 355 & 26 & 28 \\
Seine-Normandie & 69 & 110 & 19 & 19 \\
Rhin-Meuse & 34 & 50 & 46 & 42 \\
\hline
\end{tabular}

Source : SCEES (RGA 88 et STRU 95).
L'évolution de ce taux est liée au système d'irrigation pratiqué. L'augmentation d'un point de pourcentage depuis 1988, tandis que la superficie cultivée par les irrigants passe de 4,1 à 5,6 millions d'hectares, montre que le développement extensif des divers systèmes d'irrigation ne s'accompagne pas d'une intensification générale de ces systèmes.

Les évolutions régionales et départementales du taux sont plus variées: 5 régions connaissent une augmentation de leur taux comprise entre 3 et 6 points et une région connaît une diminution de 4 points. Sur les 26 départements renseignés, le taux augmente de 5 à 10 points dans 7 départements et diminue de 13 points et 6 points dans deux autres.

Comme en 1988, les taux de 1995 varient fortement d'une entité à l'autre (de 12 à $44 \%$ entre les 15 régions et de 9 à $65 \%$ entre les 26 départements renseignés).

La variation relativement faible des taux entre 1988 et 1995 semble apporter la preuve d'une assez grande stabilité des systèmes d'irrigation pratiqués localement, que le niveau de découpage spatial de l'information disponible ne permet pas d'aborder de façon plus précise.

Ces systèmes semblent s'être adaptés à la nouvelle politique agricole commune, et probablement aussi à la disponibilité de la ressource, en ajustant leur taux d'irrigation interne à la hausse ou à la baisse dans une plage de variation qu'il est difficile de connaître mais qui devrait être au moins équivalente à celle qui est constatée en département.
Carte 4. - Superficies régionales en sec et en irrigué chez les irrigants (1988-1995).

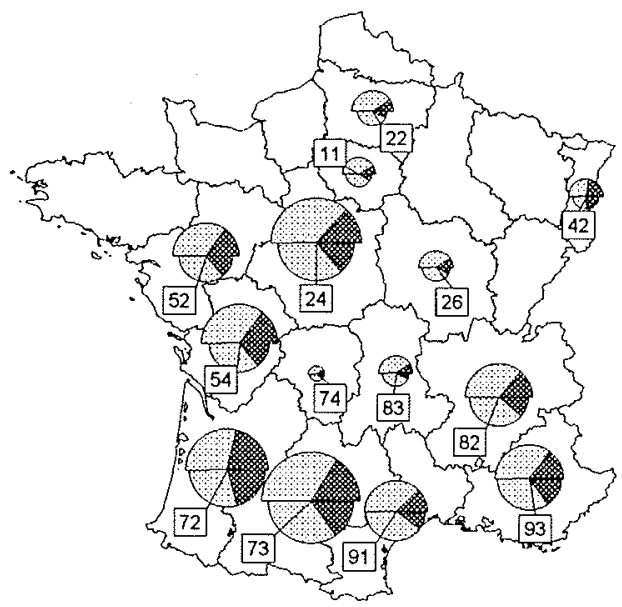

SAU \& IRRIGATION (1995)

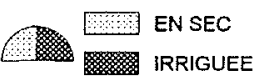

SAU \& IRRIGATION (1988)

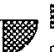

IRRIGUEE

EN SEC

15 régions renseignées

Source : SCEES (RGA 88 et STRU 95)
Tableau 10. - Hectares irrigués et taux d'irrigation interne par région (1988-1995).

\begin{tabular}{|c|c|c|c|c|}
\hline \multirow{2}{*}{ Rég. } & \multicolumn{2}{|c|}{1 000 ha irrigués } & \multicolumn{2}{c|}{$\%$ (SAU \& I) } \\
\cline { 2 - 5 } & 1988 & 1995 & 1988 & 1995 \\
\hline 73 & 210 & 299 & 30 & 33 \\
\hline 72 & 230 & 270 & 42 & 42 \\
\hline 24 & 150 & 206 & 27 & 26 \\
\hline 54 & 99 & 166 & 28 & 30 \\
\hline 93 & 116 & 129 & 30 & 30 \\
\hline 52 & 76 & 123 & 28 & 30 \\
\hline 82 & 77 & 116 & 23 & 28 \\
\hline 91 & 71 & 92 & 21 & 25 \\
\hline 42 & 34 & 50 & 48 & 44 \\
\hline 22 & 13 & 29 & 15 & 20 \\
\hline 83 & 13 & 23 & 17 & 20 \\
\hline 26 & 14 & 22 & 18 & 19 \\
\hline 11 & 13 & 19 & 19 & 19 \\
\hline 94 & 11 & 12 & 26 & 27 \\
\hline 74 & 2,1 & 3,2 & 10 & 12 \\
\hline$E n s$. & 1125 & 1558 & 29 & 30 \\
\hline
\end{tabular}

Source : SCEES (RGA 88 et STRU 95). 


\section{IRRIGATION DE NOUVELLES CUL- TURES}

Le développement de l'irrigation entre 1988 et 1995 se situe dans un contexte de diminution de $9 \%$ et de $20 \%$ des superficies cultivées respectivement en céréales et en maïs

Tableau 11. - Principales cultures irriguées (1988-1995).

\begin{tabular}{|l|r|r|r|r|}
\hline \multirow{2}{*}{ Cultures } & \multicolumn{2}{|c|}{ Irrigation 95 } & \multicolumn{2}{c|}{$\begin{array}{c}\text { Evolution } \\
\text { (100 en 88) }\end{array}$} \\
\cline { 2 - 5 } & 1 1000 ha & Tx & irrigué & cultivé \\
\hline Toutes cultures & 1620 & 5,7 & 141 & 99 \\
\hline Groupe A & 1107 & 8,1 & 146 & 97 \\
\hline Céréales & 787 & 9,5 & $126^{*}$ & 91 \\
Maïs & 705 & 44 & 123 & 80 \\
Blé dur & 11 & 4,8 & 130 & 82 \\
Autres & 71 & 1,1 & $161^{*}$ & 94 \\
Fourrages & 148 & 3,1 & 158 & 108 \\
Protéagineux & 84 & 14 & nd & 107 \\
Soja & 88 & 80 & 234 & 124 \\
\hline Groupe B & 459 & 3,9 & 125 & 90 \\
\hline Légumes frais & 144 & 53 & $151^{*}$ & 107 \\
Vergers... & 139 & 56 & $114^{*}$ & 107 \\
Betterave & 44 & 9,2 & 164 & 110 \\
Prairies & 43 & 0,5 & 90 & 86 \\
Pommes de terre & 39 & 23 & 238 & 115 \\
Vigne & 25 & 3,0 & 220 & 95 \\
Tournesol & 25 & 2,6 & 52 & 102 \\
\hline
\end{tabular}

* Calculé à partir des estimations pour 1988 qui figurent dans l'étude du SCEES

Source: SCEES (RGA 88 et STRU 95).

Carte 5. - Culture et irrigation du maïs (1988-1995).
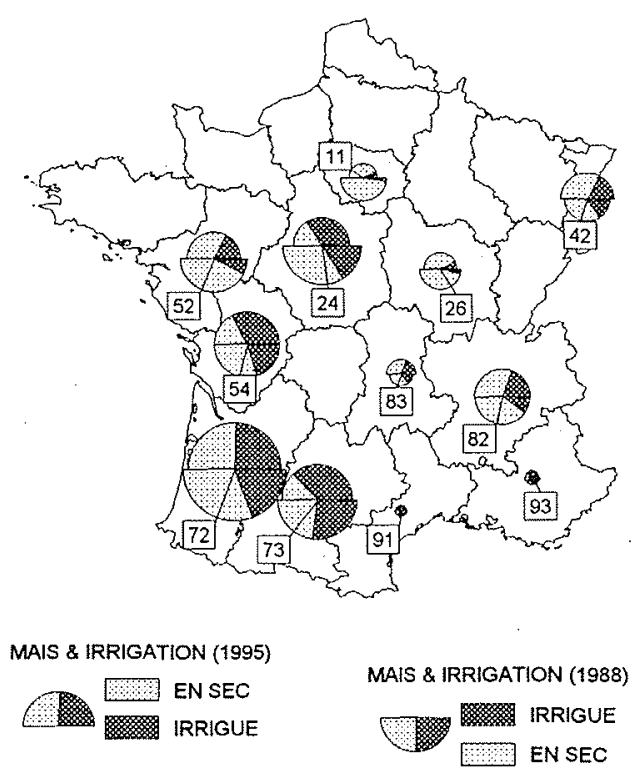

12 régions renseignées

Source : SCEES (RGA 88 et STRU 95) (grain et semences), comme le montre la dernière colonne du tableau 11 .

Les cultures du groupe A recoupent à peu près les cultures sur terres arables qui bénéficient des aides compensatoires de la PAC depuis 1993. L'irrigation du soja s'est imposée, avec un taux d'irrigation de $80 \%$. Celle des protéagineux est passée de quelques milliers d'hectares en 1988 à plus de 80000 ha en 1995 (60000 ha en 1993). L'irrigation des fourrages se développe. Elle semble plafonner sur les autres céréales.

L'irrigation des légumes frais augmente en continu ( 95000 ha en 1988, 126000 ha en 1993). Celle des pommes de terre de plein champ a plus que doublé sur la période tandis que celle du tournesol a été divisée par deux.

\section{VII 圈 44\% DU MAIS GRAIN EST IRRI- GUÉ}

La culture du maïs-grain et du maïs-semence a diminué de 393000 ha entre 1988 et 1995 , notamment dans les principales régions de production, sauf en Poitou-Charente, Alsace et Rhône-Alpes.

Le taux d'irrigation global est passé 29 à $44 \%$. La superficie cultivée en maïs des exploitations équipées pour l'irrigation a augmenté de $10 \%$ entre 1988 et 1995, le taux d'irrigation du maïs de ces exploitations passant de $73 \%$ à $82 \%$. La proximité du taux plafond d'irrigation en interne et la diminution de 4000 ha irrigués entre 1993 et 1995 pourraient être les signes d'un palier autour de 700000 ha irrigués.

Tableau 12. - Irrigation du maïs (1988-1995).

\begin{tabular}{|c|c|c|c|c|}
\hline \multirow[t]{2}{*}{ Territoires } & \multicolumn{2}{|c|}{ Irrigation 95} & \multicolumn{2}{|c|}{ Taux global } \\
\hline & 1000 ha & 100 en 88 & 1988 & 1995 \\
\hline \multirow[t]{2}{*}{ France } & 705 & 123 & 89 & 44 \\
\hline & \multicolumn{4}{|c|}{ Données par bassin } \\
\hline$A-G$ & 398 & 112 & 45 & 59 \\
\hline$L-B$ & 182 & 139 & 21 & 39 \\
\hline$R-M-C$ & 60 & 152 & 20 & 31 \\
\hline$R-M$ & 41 & 158 & 27 & 33 \\
\hline$S-N$ & 24 & 103 & 8,2 & 18 \\
\hline Ens. & 705 & 123 & 29 & 44 \\
\hline \multicolumn{5}{|c|}{ Données par région } \\
\hline 72 & 173 & 103 & 39 & 49 \\
\hline 73 & 153 & 111 & 54 & 75 \\
\hline 54 & 116 & 162 & 40 & 66 \\
\hline 24 & 91 & 111 & 33 & 69 \\
\hline 82 & 49 & 189 & 21 & 38 \\
\hline 52 & 43 & 151 & 15 & 36 \\
\hline 42 & 41 & 158 & 31 & 36 \\
\hline 83 & 13 & 199 & 28 & 36 \\
\hline 26 & 6,4 & 135 & 6,7 & 14 \\
\hline 93 & 6,3 & 73 & 81 & 87 \\
\hline 11 & 4,4 & 109 & 4,7 & 13 \\
\hline 91 & 3,8 & 74 & 73 & 81 \\
\hline Ens. & 699 & 123 & 33 & 52 \\
\hline
\end{tabular}

Source: SCEES (RGA 88 et STRU 95). 
VIII $62 \%$ DES IRRIGATIONS SONT ÉLIGIBLES AUX AIDES DE LA POLITIQUE AGRICOLE COMMUNE (1995)

Ce taux est plus élevé que le taux de superficie agricole utilisée concernée par les aides $(40 \%)$. Les variations locales de ces deux taux s'expliquent surtout par la présence de cultures de légumes frais et de vergers, irrigués ou non.

\section{Une analyse provisoire du fichier de l'ONIC}

Les données présentées ici reposent sur les déclarations des agriculteurs centralisées par l'Office national interprofessionnel des céreales pour liquider les aides de la PAC de la campagne agricole 1995. Elles sont en cours de validation par le SCEES dans le cadre de la mise en place d'une statistique administrative issue de la gestion des aides. Elles sont proches, sans les recouper, des données publiées [3]. Les données parcellaires dispo nibles sont regroupées ici par commune de localisation des parcelles, et non par commune du siege de l'exploitation.

Carte 6. - Irrigation des cultures annuelles aidées par la PAC et autres irrigations (1995).

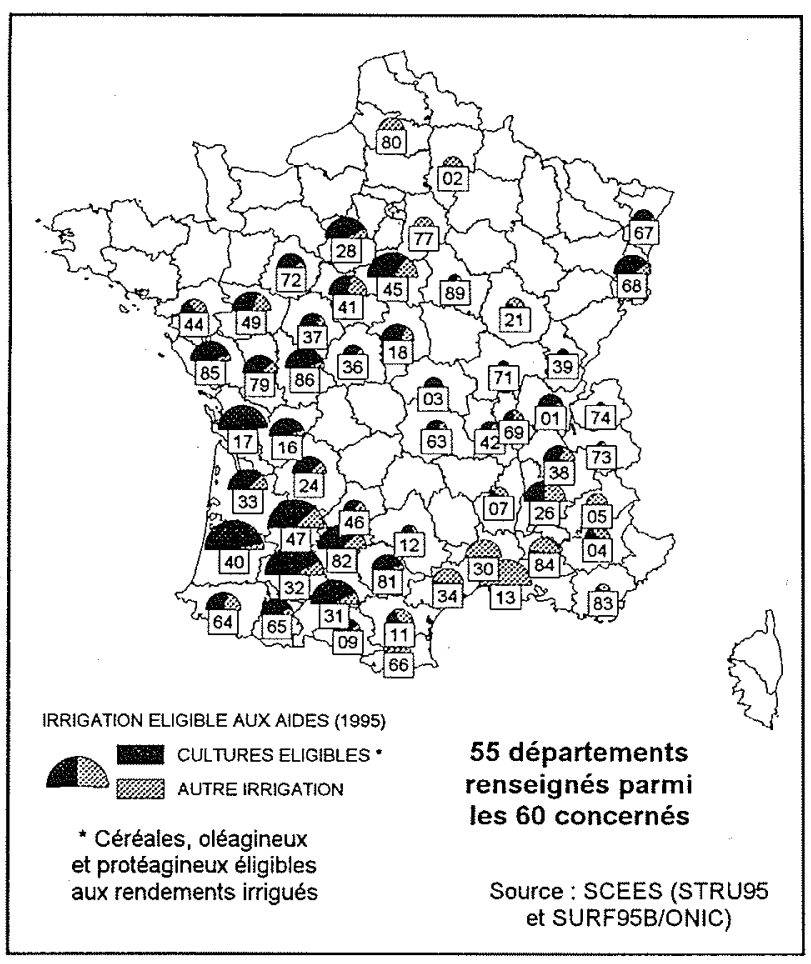

Tableau 13. - Superficies cultivées et superficies irriguées éligibles (1995).

\begin{tabular}{|l|r|r|r|r|}
\hline \multicolumn{1}{|c|}{ Territoire } & 1 000 ha & $\%$ SAU & 1 000 ha & $\%$ SI \\
\hline France & 11449 & 40 & 1012 & 62 \\
\hline Adour-Garonne & 2033 & 37 & 548 & 83 \\
Rhône-Médit.-C. & 986 & 24 & 82 & 23 \\
Loire-Bretagne & 3890 & 40 & 299 & 72 \\
Rhin-Meuse & 646 & 45 & 42 & 82 \\
Seine-Normandie & 375 & 53 & 41 & 37 \\
Artois-Picardie & 709 & 50 & 0,1 & 0,5 \\
& & & & \\
lle-de-France & 412 & 69 & 1,8 & 9,2 \\
Picardie & 753 & 55 & 0,7 & 2,3 \\
Centre & 495 & 62 & 151 & 73 \\
Bourgogne & 731 & 41 & 11 & 52 \\
Alsace & 180 & 53 & 42 & 83 \\
Franche-Comté & 170 & 25 & 3,4 & 69 \\
Pays de la Loire & 878 & 40 & 88 & 72 \\
Poitou-Charentes & 931 & 52 & 150 & 91 \\
Aquitaine & 592 & 39 & 218 & 81 \\
Midi-Pyrénées & 955 & 40 & 243 & 81 \\
Rhône-Alpes & 405 & 26 & 65 & 56 \\
Auvergne & 256 & 17 & 19 & 81 \\
Languedoc-R. & 138 & 14 & 9,5 & 10 \\
P.A.C.A. & 103 & 16 & 8,2 & 6,4 \\
\hline
\end{tabular}

Source: SCEES (STRU95 et SURF95B/ONIC).

Tableau 14. - Superficies irriguées éligibles par département (1995).

\begin{tabular}{|c|c|c|c|c|c|}
\hline Dép. & 1000 ha & $\% \mathrm{SI}$ & Dép. & $1000 \mathrm{ha}$ & $\% S I$ \\
\hline 01 & 14 & 88 & 42 & 5,4 & 64 \\
\hline 02 & 0,6 & 5,5 & 44 & 7,1 & 38 \\
\hline 03 & 11 & 100 & 45 & 47 & 71 \\
\hline 04 & 5,3 & 32 & 46 & 7,8 & 62 \\
\hline 05 & 0,1 & 0,6 & 47 & 61 & 75 \\
\hline 07 & 1,8 & 21 & 49 & 24 & 62 \\
\hline 09 & 13 & 83 & 63 & 6,5 & 61 \\
\hline 11 & 7,1 & 36 & 64 & 19 & 60 \\
\hline 12 & 4,7 & 70 & 65 & 23 & 87 \\
\hline 13 & 0,3 & 0,4 & 66 & 0,0 & 0,1 \\
\hline 16 & 29 & 88 & 67 & 12 & 93 \\
\hline 17 & 62 & 99 & 68 & 30 & 81 \\
\hline 18 & 21 & 71 & 69 & 5,4 & 49 \\
\hline 21 & 1,3 & 13 & 71 & 2,3 & 84 \\
\hline 24 & 23 & 74 & 72 & 18 & 84 \\
\hline 26 & 22 & 50 & 73 & 0,3 & 17 \\
\hline 28 & 41 & 86 & 74 & 0,2 & 16 \\
\hline 30 & 1,0 & 3,1 & 77 & 0,0 & 0,1 \\
\hline 31 & 50 & 83 & 79 & 24 & 76 \\
\hline 32 & 77 & 84 & 80 & 0,1 & 0,7 \\
\hline 33 & 30 & 75 & 81 & 23 & 90 \\
\hline 34 & 1,4 & 5,9 & 82 & 44 & 73 \\
\hline 36 & 8,7 & 79 & 83 & 1,3 & 24 \\
\hline 37 & 13 & 76 & 84 & 1,2 & 4,9 \\
\hline 38 & 16 & 63 & 85 & 37 & 90 \\
\hline 39 & 2,9 & 77 & 86 & 36 & 91 \\
\hline 40 & 85 & 94 & 89 & 3,1 & 68 \\
\hline 41 & 21 & 55 & Ens & 1001 & 65 \\
\hline
\end{tabular}

Source: SCEES (STRU95 et SURF95B/ONIC). 


\section{RÉFÉRENCES}

11] Etude $n^{\circ} 13$ du SCEES. Irrigation et drainage en France, décembre 1991.

12| Développer un système d'observation statistique de l'usage et de l'origine de l'eau d'irrigation (bulletin du CGGREF $\left.n^{\circ} 43\right)$.

[3] Paiements compensatoires aux superficies cultivées, 1995 , ONIC-SIDO-Agreste (La statistique agricole).

\section{EN GUISE DE CONCLUSION}

L'analyse des données de la statistique agricole sur l'irrigation depuis 1988 illustre l'augmentation notable des superficies irriguées et la part importante occupée par les cultures irriguées éligibles aux aides de la politique agricole commune.

Lorsque le développement local des prélèvements pour l'irrigation affecte le débit estival des cours d'eau au point d'entrainer de façon chronique la suspension provisoire ou l'interdiction totale des arrosages en cours de campagne, ce développement joue clairement contre l'objectif même de l'irrigation et prive les investissements consentis de toute signification.

Pour éviter de tels errements, la loi a permis d'abaisser dans certaines zones, dites « de répartition des eaux », le seuil de capacité des prélèvements individuels ou collectifs soumis à autorisation, qui passe ainsi de $80 \mathrm{~m}^{3} / \mathrm{h}$ à $8 \mathrm{~m}^{3} / \mathrm{h}$ (décret du 29 avril 1994 pris en application de l'article 8-20 de la loi sur l'eau du 3 janvier 1992). Le bassin AdourGaromne est principalement visé par ces dispositions.

Dans ce contexte, il est indispensable de bien connaître les pratiques en matière d'irrigation. Les recensements et les enquêtes actuelles de la statistique agricole, en dehors des enquêtes sur les pratiques culturales en terrains irrigués de 1986 et 1994, sont axés sur les superficies irriguées et sont donc mal adaptés à la connaissance quantitative des prélèvements d'eau pour l'irrigation.

\section{DÉ DELOPPER LE COMPTAGE DE L'EAU}

Une bonne connaissance des usages de l'eau, tant au niveau individuel que collectif, ne peut se satisfaire d'une évaluation forfaitaire des besoins en eau et des consommations effectives des cultures irriguées sur la base de ratios techniques issus de débats d'experts.

La faisabilité et l'utilité d'une gestion statistique des comptages et des données administratives fait actuellement l'objet d'une étude préalable qui a débuté sous la forme d'une enquête approfondie auprès des services compétents du bassin Adour-Garonne.

Le dispositif statistique devra éviter toute confusion fonctionnelle avec le système source, constitué par le dispositif informatisé d'acquisition et de suivi des données nominatives par les services de l'Etat chargés de la police de l'eau, actuellement en cours de refonte.

L'enquête a montré que l'adhésion à un réseau collectif qui prélève dans un cours d'eau n'apporte pas plus de garantie de ressource à l'irrigant que le prélèvement individuel dans le même cours d'eau lorsque celui-ci est de plus en plus sollicité.

Pour les deux types de prélèvement dans une telle situation, l'autorisation est en effet soumise à la signature d'une convention de restitution du débit prélevé avec l'organisme gestionnaire du soutien d'étiage, si toutefois un tel dispositif a pu être mis en place.

\section{XI 橉 UNE STATISTIQUE PUBLIQUE DE L'EAU D'IRRIGATION ?}

La mise en cuvre de systèmes de comptage sur tous les prélèvements constitue l'enjeu majeur du nouveau contexte réglementaire de gestion de la ressource, afin d'apporter aux irrigants, aux gestionnaires des ressources, aux agences de l'eau, aux services de l'Etat et à tous ses partenaires une perspective commune, axée sur « l'économie » de l'eau utilisée.

Si cette condition est remplie, il semble possible d'envisager que l'appareil statistique de l'Etat mette en place un dispositif de statistique publique des débits de prélèvement des volumes stockés et des volumes prélevés afin d'apporter sa contribution aux prises de décision de toutes sortes dans un domaine sensible. 